\title{
CircP4HB regulates ferroptosis via SLC7A11-mediated glutathione synthesis in lung adenocarcinoma
}

\author{
Chun-Feng Pan ${ }^{1 \#}$, Ke Wei ${ }^{1 \#}$, Zi-Jian Ma ${ }^{2 \#}$, Yao-Zhou He ${ }^{3}$, Jing-Jing Huang ${ }^{1}$, Zi-Zhang Guo ${ }^{1}$, \\ Zhi-Peng Chen ${ }^{1}$, Martin P. Barr ${ }^{4}$, Rodney E. Shackelford ${ }^{5}$, Yang Xia ${ }^{1}$, Jun Wang ${ }^{1}$
}

${ }^{1}$ Department of Thoracic Surgery, The First Affiliated Hospital with Nanjing Medical University, Nanjing, China; ${ }^{2}$ Department of Thoracic Surgery, Nanjing First Hospital, Nanjing Medical University, Nanjing, China; ${ }^{3}$ Department of Oncology, The First Affiliated Hospital with Nanjing Medical University, Nanjing, China; ${ }^{4}$ Thoracic Oncology Research Group, School of Medicine, Trinity Translational Medicine Institute, Trinity Centre for Health Sciences, St. James's Hospital and Trinity College Dublin, Dublin, Ireland; ${ }^{5}$ Department of Pathology, LSU Health Shreveport, Shreveport, LA, USA

Contributions: (I) Conception and design: CF Pan, Y Xia, J Wang; (II) Administrative support: J Wang; (III) Provision of study materials or patients: CF Pan, K Wei, ZP Chen, JJ Huang, J Wang; (IV) Collection and assembly of data: CF Pan, K Wei, ZJ Ma; (V) Data analysis and interpretation: K Wei, YZ He, ZZ Guo, Y Xia; (VI) Manuscript writing: All authors; (VII) Final approval of manuscript: All authors.

\#These authors contributed equally to this work.

Correspondence to: Jun Wang; Yang Xia. No. 300 Guangzhou Road, Nanjing 210009, China. Email: drwangjun@njmu.edu.cn; xiayang-1019@njmu.edu.cn.

Background: Circular ribonucleic acids (circRNAs) play a key role in the development of different types of cancer. Ferroptosis is a type of programmed cell death that contributes to cancer progression. However, the role of circRNAs in lung adenocarcinoma (LUAD) ferroptosis remains unclear.

Methods: The gene expression levels of circRNA P4HB (circP4HB), microRNA-1184 (miR-1184) and Solute carrier family 7 member 11 (Slc7a11), also known as Xct were detected using quantitative real-time polymerase chain reaction (qRT-PCR). Ferroptosis of established LUAD cells was induced by erastin. Cell viability was examined via Cell Counting Kit 8 assays. Ferroptosis was evaluated by malondialdehyde (MDA), Prostaglandin-endoperoxide Synthase 2 (Ptgs2), lipid reactive oxygen species (lipid ROS), and JC-1 detection. The mechanism of circP4HB/miR-1184/SLC7A11 was investigated by luciferase reporter assays, RNA immunoprecipitation, RNA pull-down, and western blot assays. A functional for circP4HB in vivo was determined using xenograft nude mice models.

Results: CircP4HB expression levels were increased in LUAD. It triggered glutathione (GSH) synthesis and, therefore protected LUAD cells from ferroptosis induced by erastin. CircP4HB may function as a competing endogenous RNA by modulating miR-1184 to regulate SLC7A11. CircP4HB inhibited ferroptosis by regulating miR-1184/ SLC7A11-mediated GSH synthesis. In vivo, overexpression of circP4HB promoted tumor growth and inhibited ferroptosis.

Conclusions: The circRNA, circP4HB acts as a novel ferroptosis suppressor in LUAD. Furthermore, circP4HB protects LUAD from ferroptosis via modulation of the miR-1184/SLC7A11 axis. Our findings identified circ $\mathrm{P} 4 \mathrm{HB}$ as a novel biomarker in LUAD and warrants further investigation in the early diagnosis and treatment of LUAD.

Keywords: Circular ribonucleic acids (circRNAs); miR-1184; SLC7A11; lung adenocarcinoma (LUAD); glutathione (GSH)

Submitted Jan 16, 2022. Accepted for publication Feb 25, 2022.

doi: $10.21037 /$ tlcr-22-138

View this article at: https://dx.doi.org/10.21037/tlcr-22-138 


\section{Introduction}

Lung adenocarcinoma (LUAD) is a common histologic variant of lung cancer, and a leading cause of cancerrelated mortality (1). The incidence of LUAD has shown a significant upward trend (1). Despite continuous improvement in treatment options, the survival rate of patients with LUAD remains low poor (2). Tumor size is one of the most important factors in determining staging and treatment, which greatly affects the patients' prognosis (3). Tumor size is mainly due to the uncontrolled tumor cell growth. An imbalance between tumor growth and death is an important factor in tumor development. Recent studies have reported that the induction of tumor cell ferroptosis can significantly inhibit tumor growth (4-7).

Ferroptosis is a form of programmed cell death (8), and its dysfunction has been implicated in various diseases, particularly cancer (9). Ferroptosis involves many biological processes, such as iron metabolism (10), lipid metabolism (11), oxidative stress (12), and glutathione (GSH) (13), which are related to cancer progression, and provides a source for cancer cell growth. Thus, it is important to explore the abnormal metabolism mechanism related to cancer ferroptosis in order to develop more effective therapeutic strategies.

Circular ribonucleic acids (circRNAs) are reliable biomarkers in diagnosis and prognosis, and their role in the regulation of cancer metastasis and growth has become an area of significant research interest in recent years $(14,15)$. Currently, research on the function of circRNAs in ferroptosis has been limited. While circRNAs are important regulators in cancer cellular metabolism (16), the role of circRNAs in abnormal metabolism related to cancer ferroptosis has not been fully elucidated.

In the current research, we identified a novel ferroptosis suppressor circRNA derived from the P4HB gene (hsa circ_0046263, circP4HB). This study, to our knowledge, is the first to show that circ $\mathrm{P} 4 \mathrm{HB}$ protected LUAD from ferroptosis via Solute carrier family 7 member 11 (SLC7A11)-mediated GSH synthesis. Our findings provide new insights into the mechanisms of LUAD progression. We present the following article in accordance with the ARRIVE reporting checklist (available at https:// tlcr.amegroups.com/article/view/10.21037/tlcr-22-138/rc).

\section{Methods}

\section{Cell lines}

All cell lines were obtained from the Chinese Academy of Sciences (Shanghai, China) and included the following; H1299, A549, SPCA1 and H358 lung adenocarcinoma cell lines. The human bronchial epithelial cell line, 16HBE, was also used. Cells were cultured with RPMI 1640 medium (Corning, NY, USA) containing $10 \%$ fetal bovine serum (FBS) and $1 \%$ penicillin/streptomycin at $37^{\circ} \mathrm{C}$ in humidified air containing $5 \% \mathrm{CO}_{2}$.

\section{Transfections}

H1299 and SPCA1 cell lines were transfected with lentiviral vectors (GenePharma, Shanghai, China). Small interfering ribonucleic acids (siRNAs) for suppressing Slc7a11 were purchased from GenePharma (Shanghai, PR China). Cells were transfected with oligonucleotides microRNA-1184 (miR-1184) or a negative control (NC) (miR-control), (GenePharma, Shanghai, PR China) and transfected using Lipofectamine 3000 Reagent (Invitrogen, CA, USA).

\section{Clinical samples}

Tumor and para-tumor tissues ( $\geq 2 \mathrm{~cm}$ from the tumor margin) were derived from 50 patients with LUAD at The First Affiliated Hospital with Nanjing Medical University. The tissue fragments were immediately frozen in liquid nitrogen for next use. The present study was approved by the Institutional Ethics Committee of the First Affiliated Hospital with Nanjing Medical University (No. 2019SFRA-082), and written informed consent was obtained from each participant. The study was conducted in accordance with the Declaration of Helsinki (as revised in 2013)

\section{$R N A$ isolation and quantitative real-time polymerase chain reaction ( $q R T-P C R)$}

Total RNA of cells and clinical samples were isolated using TRIzol Reagent (Invitrogen, CA, USA) and reverse transcribed into cDNA using a PrimeScript RT reagent Kit (Takara, Beijing, PR China) according to the manufacturer's 
protocol. qRT-PCR was carried out in accordance with our previous study (17). Primers were as follows: circP4HB, forward 5'-ACCATTTGGGATCACTTCCA-3'; reverse 5'-CTTCTTCAGCCAGTTCACGA-3'; miR-1184, forward 5'-CCTGCAGCGACTTGATGGC-3'; reverse 5'-GAACATGTCTGCGTATCTC-3'; Slc7a11, forward 5'-TCATTGGAGCAGGAATCTTCA-3'; reverse 5'-TTCAGCATAAGACAAAGCTCCA-3'; Ptgs2, forward 5'-TGGTCTGGTGCCTGGTCTGATG-3'; reverse 5'-CCTGCTTGTCTGGAACAACTGCTC-3'; Gclc, forward 5'-AACACAGACCCAACCCAGAG-3'; reverse 5'-CCGCATCTTCTGGAAATGTT-3'; Gclm, forward 5'-GCCACCAGATTTGACTGCCTTT-3'; reverse 5'-CAGGGATGCTTTCTTGAAGAGCTT-3'; Gsr, forward 5'-ATTGGCTGTGATGAGATGCT-3'; reverse 5'-GGTAGGATGAATGGCAACTG-3'; Gss, forward 5'-CCAGCGTGCCATAGAGAATGAGC-3'; reverse 5 '-AGCCTTCGGTCTTGGTCCAGAG-3'; Nqo1, forward 5'-CAGATCCTGGAAGGATGGAA-3'; reverse 5'-TCTGGTTGTCAGCTGGAATG-3'.

\section{Cell viability}

Cell viability was determined using the cell counting kit 8 (CCK-8) assay to examine the effect of erastin or circP4HB on cell viability. Transfected cells $\left(6 \times 10^{3}\right)$ were seeded per well in a 96-well plate and then stained with $10 \mu \mathrm{L}$ of CCK8 solution (Beyotime, Shanghai, China) for $4 \mathrm{~h}$. Absorbance was read at $450 \mathrm{~nm}$ using a photometer (Thermo Scientific, Rockford, IL, USA).

\section{GSH, GSH/oxidized glutathione disulfide (GSSG) detection}

SPCA1 or H1299 cells after transfection were cultured and harvest (10 $\mathrm{mg}$ of cell pellet) and GSH level were measured by GSH detection assay kit (Beyotime, Shanghai, China) according to the manufacturer's instructions. Transfected SPCA1 or H1299 cells $\left(6 \times 10^{4}\right.$ cells/well) were seeded into 96-well white-walled multi-well luminometer plates and cultured for $24 \mathrm{~h}$. The GSH/GSSG ratio was detected using a GSH/GSSG Ratio Detection Assay kit (Abcam, CA, USA), following the manufacturer's protocols.

\section{Malondialdehyde quantification}

A total of $1 \times 10^{6}$ SPCA1 and H1299 cells transfected with lentiviral vectors were harvested and ultrasonicated.
Malondialdehyde (MDA) levels were measured in the lysates using the Lipid Peroxidation Assay Kit (S0131, Beyotime, Shanghai, China) following the kit's protocol. Absorbance was measured at $532 \mathrm{~nm}$.

\section{Lipid ROS staining}

Lipid peroxidation of SPCA1 and H1299 cell lines was measured using C11-BODIPY 581/591 (Thermo Fisher Scientific). In brief, C11-BODIPY581/591 was added to the supernatant of the cell culture medium at a final concentration of $5 \mu \mathrm{mol} / \mathrm{L}$. After incubation at $37{ }^{\circ} \mathrm{C}$ for $30 \mathrm{~min}$ in the dark and washing with PBS, cells were photographed using a fluorescence microscope (Olympus, Tokyo, Japan).

\section{$\mathcal{F C}-1$ staining}

A JC-1 fluorescence probe kit (Beyotime, Shanghai, China) was used to detect the mitochondrial membrane potential of the cells. Cells were added with JC-1 solution in the culture medium, and incubated for $20 \mathrm{~min}$, and then washed with the JC-1 buffer solution. Photographs were taken using a fluorescence microscope (Olympus). The $\mathrm{red} /$ green fluorescence intensity ratio was measured using Image J software (NIH, USA) which indicated the degree of mitochondrial damage.

\section{Luciferase reporter assays}

The mutant circP4HB 3'-untranslated region (3'-UTR) with the predicted miR-1184 binding sites were cloned into the luciferase reporter vector psi-CHECK-2 (Geneseed, Shanghai, China). The 3'-UTR sequence or the mutant sequence of Slc7a11 was inserted into the promoter vector. Cells were transfected with luciferase reporter vectors and the miR-1184 mimic via using Lipofectamine 3000 (Invitrogen). Activity of luciferase was read using the Victor 1420 Multilabel Counter (Wallac, Finland) as per the manufacturer's instructions.

\section{RNA immunoprecipitation (RIP)}

RIP was carried out using a Magna RIP Kit (Millipore, Billerica, USA) according to the manufacturer's instructions. Anti-Ago2 antibody was applied, and negative control immunoglobulin G (IgG; Millipore, USA) was employed as the control. The transfected H1299 and 
SPCA1 cells were lysed using RNA lysis reagent and then maintained with anti-Ago2 antibody $(5 \mu \mathrm{g})$ or $\mathrm{IgG}$ overnight at $4{ }^{\circ} \mathrm{C}$. Magnetic beads $(40 \mu \mathrm{L})$ were added and cultured for $24 \mathrm{~h}$. The RNA/bead complexes were washed and then resuspended in Proteinase K buffer. Next, the immunoprecipitated RNA was purified and examined by using qRT-PCR.

\section{RNA pull-down assays}

The random oligo probe and biotin labelled probe specifically targeting circP4HB (RiboBio, Guangzhou, China) were obtained and were incubated with M280 streptavidin Dynabeads (Invitrogen, USA) for $2 \mathrm{~h}$ at room temperature. Lysates of H1299 and SPCA1 cells were incubated overnight with the probe/bead complexes at $4{ }^{\circ} \mathrm{C}$. Then, the circP4HB/ miRNA/bead complexes were washed and eluted from the beads. Then, the enrichment of circP4HB and related miRNAs was examined using qRT-PCR.

\section{Fluorescence in situ bybridization (FISH)}

FISH assays was carried out using the Fluorescent In Situ Hybridization Kit (GenePharma, Shanghai, China) in accordance with the manufacturer's instructions. A total of 3000 cells were seeded into confocal dishes and fixed. Cy3labelled circP4HB and FAM-labelled miR-1184 probes (GenePharma, Shanghai, China) were added to the cells. Cell nuclei were stained with 4,6-diamidino-2-phenylindole. Fluorescence was excited and imaged using by a confocal laser scanning microscope (Carl Zeiss, Germany).

\section{Western blotting}

Protein expression was determined using western blot analysis as previously reported. We adopted the same experimental protocol as that in our previous study (17). A total of $30 \mu \mathrm{g}$ proteins were analyzed using $10 \%$ SDSPAGE gel and transferred to polyvinylidene fluoride (PVDF) membranes (Millipore, MA, USA). Primary antibody for SLC7A11 and Goat anti-Rabbit IgG (H+L)HRP was obtained from Cell Signaling Technology (1: 1,000, \#12691, Danvers, MA).

\section{Immunobistochemistry analysis}

Tissue samples were fixed with formalin, paraffin embedded, and sliced into tissue sections ( $5-\mu \mathrm{m}$ thick). After antigen recovery, the sections were incubated with $3 \% \mathrm{H}_{2} \mathrm{O}_{2}$ for $10 \mathrm{~min}$, subsequently blocked at room temperature for $1 \mathrm{~h}$ in $5 \%$ NGS. The sections were incubated with primary antibodies for SLC7A11 at $4{ }^{\circ} \mathrm{C}$ overnight. The cell nuclei were counterstained with hematoxylin. After that, an HRPDAB system (Beyotime, Shanghai, China) was applied and representative images were obtained via using Olympus microscope (Olympus, Tokyo, Japan).

\section{Animal studies}

Two-month-old male nude mice housed and maintained under specific-pathogen-free (SPF) conditions in an animal facility were divided into 4 groups ( $\mathrm{n}=6$ per group, randomly), and injected subcutaneously into the dorsal flanks with $1 \times 10^{6}$ SPCA1 cells transfected with lentivirus for circP4HB (circP4HB) or its negative control (NC) and maintained with erastin or Dimethyl sulfoxide (DMSO). No blinding experimental method was used in this study. Tumor sizes were measured every 3 days. The mice were sacrificed 4 weeks later and tissue samples were collected. The following formula was used to calculate tumor volume: tumor volume $=($ width + length $) / 2 \times$ width $\times$ length $\times$ 0.5236 . The animal experiments were performed according to the guidelines of the Institutional Animal Care and Use Committee of Nanjing Medical University (Nanjing, China) and protocols were approved by the Institutional Animal Care and Use Committee of Nanjing Medical University (No. IACUC:2009054).

\section{Statistical analysis}

Data are representative of the mean \pm standard error of the mean (SEM). The student's unpaired $t$-test and ANOVA were used for the analysis. A $\mathrm{P}$ value $<0.05$ was considered statistically significant. Statistical analysis was conducted using with STATA 12 software.

\section{Results}

\section{CircP4HB triggers GSH synthesis}

To gain further insights into the role of dysregulated circRNAs in LUAD, we re-analyzed the published data set (GSE101586, GSE112214 and GSE101684) from the Gene Expression Omnibus database to confirm circRNAs with abnormal levels between LUAD and para-tumor tissues. Among the abnormally expressed circRNAs, upregulated 
circRNAs were more common than downregulated circRNAs in these published data set. Also, previous studies have focused on that some circRNAs are upregulated in LUAD and display oncogenic functions in LUAD (18-21). Heatmap analysis showed that hsa_circ_0046263 (circP4HB), hsa_circ_0006006, hsa_circ_0057105, hsa_ circ_0057104, hsa_circ_0000520, and hsa_circ_0003528 were all simultaneously and aberrantly expressed in LUAD tissues compared to para-tumor tissues (Figure $1 A, 1 B$ ). It has been reported that circP4HB contributes into lung cancer metastasis, but its role in cell proliferation and death remains unclear; thus, we focused on circP4HB in this study.

The Cancer Genome Atlas (TCGA) data exhibited significantly higher $\mathrm{P} 4 \mathrm{HB}$ expression levels in LUAD tissue compared to normal lung tissue samples (Figure 1C). The Kaplan-Meier plotter analysis indicated that patients with high $P 4 h b$ expression levels had significantly poorer overall survival (Figure 1D). Many metabolic pathways had high P4hb levels (Figure 1E), of which the GSH metabolism pathway was most biologically active (Figure $1 F$ ). Higher levels of circP4HB in LUAD tissues were detected by qRT-PCR compared to adjacent para-tumor tissues (see Figure $1 G$ ). Based on the above data, we speculated that circ_0046263 was related to GSH metabolism. The GSH (the end-product of GSH metabolism) and the GSH/ GSSG ratio in the circP4HB overexpressing cells were significantly upregulated (Figure 1H,1I), suggesting that cells overexpressing circP4HB highly activate GSH synthesis.

\section{CircP4HB inhibits ferroptosis in LUAD cells from by promoting GSH synthesis}

As GSH metabolism plays an important role in multiple cancers $(22,23)$, and GSH depletion induces ferroptosis (13), we explored the effect of circP4HB on ferroptosis. First, to clarify whether ferroptosis is implicated in LUAD, we examined cellular ferroptosis by adding erastin to the culture media of different LUAD cell lines (A549, SPCA1, H1299, H358) and in the normal bronchial epithelial cell line, 16HBE cells. We found that erastin inhibited cell viability by inducing ferroptosis. Notably, we observed that all the tested LUAD cells became more resistant to ferroptosis than the 16HBE cells (Figure 2A). Additionally, the ferroptosis markers MDA and Ptgs2 were significantly upregulated in the all cell lines examined (Figure 2B,2C). The upregulation of circ 4 HB significantly inhibited erastin-induced cell death (Figure 2D). GSH and the GSH/GSSG ratio in the circP4HB upregulated cells were increased (Figure 2E,2F), while MDA and Ptgs2 levels were decreased (Figure 2G,2H). In addition, immunofluorescence staining of cells showed that lipid reactive oxygen species (ROS) was significantly decreased in response to erastin in cells overexpressing circP4HB, while JC-1 as significantly increased relative to erastin alone (Figure 2I,2f). Similar effects in the SPCA1 cells were observed (Figure S1). All these findings indicated that circ $\mathrm{P} 4 \mathrm{HB}$ protected LUAD cells from ferroptosis by promoting GSH synthesis.

\section{Validation of the interaction between circP4HB, miR- 1184, and SLC7A11}

As shown in Figure 3A, circP4HB was found to be located, for the most part, in the cell cytoplasm, with less expression in the nuclear compartment. CircRNAs are rich in miRNA binding sites, and acts as a "miRNA sponges", otherwise known as competing endogenous RNA (ceRNA) to relieve miRNA's inhibitory effect on its target genes, thereby upregulating the expression levels of the target genes $(15,24)$. Using bioinformatic predications (https://circinteractome. nia.nih.gov; circNet) suggested that miR-1184 is a potential target of circP4HB (Figure 3B,3C). MiR-1184 is related to the development of different cancers, and acts as a downstream molecule of several circRNAs.

To confirm the interaction between circ $\mathrm{P} 4 \mathrm{HB}$ and miR-1184, we mutated the binding site of circP4HB. The dual-luciferase reporter assay results suggested that with the loss of the binding site, circP4HB was not able to sequester miR-1184 (Figure 3D). Argonaute 2 (AGO2) has been demonstrated to be a key protein that participates in miRNA function in an RNA-induced silencing complexdependent manner (25). Thus, RIP assays were performed on the H1299 and SPCA1 cells using the AGO2 antibody, and the results suggested that circP4HB was enriched by the AGO2 antibody (Figure 3E). The RNA pull-down assays also indicated that circP4HB directly binds to miR-1184. A significant enrichment of circP4HB and miR-1184 was also observed (Figure $3 F$ ). In summary, the above findings indicated that circP4HB targeted and sponged miR-1184.

MiRNAs are small (17-24 nucleotides) non-coding RNAs, which binds to the 3'-UTR of messenger RNA (mRNA), and mediate post-transcriptional silencing of genes, many of which have now been reported to be implicated in cancer (17). Based on predictions in TargetScan and FerrDb, we found many candidates, among 


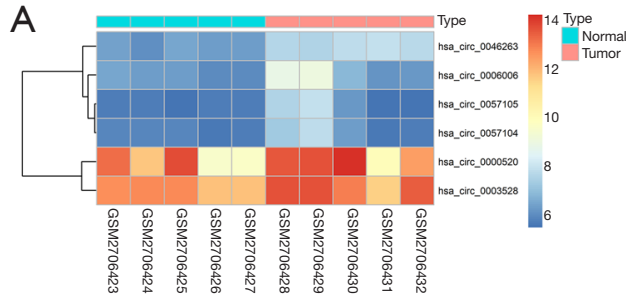

B

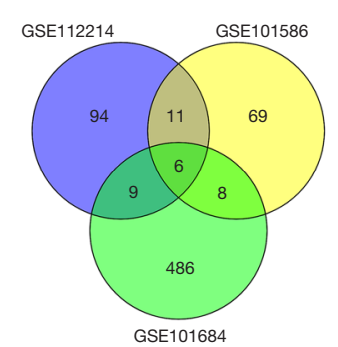

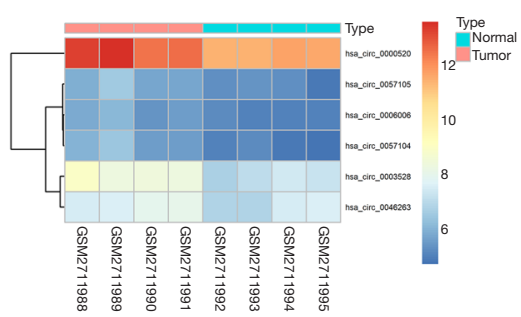

C

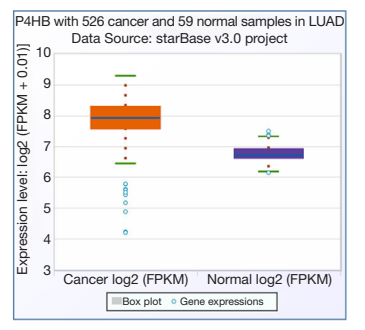

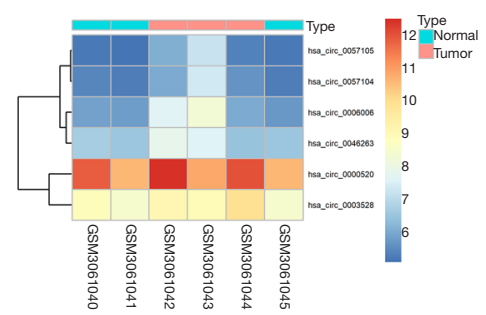

D

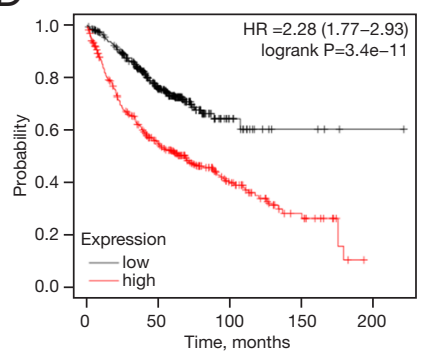

$\mathrm{F}$
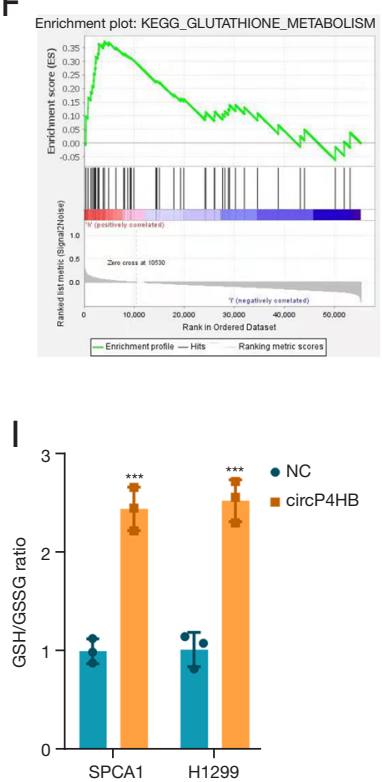

Figure 1 CircP4HB triggered GSH synthesis. (A,B) The GSE101586, GSE112214, and GSE101684 data sets were re-analyzed to identify the circRNAs that were differentially expressed between the LUAD and adjacent normal tissues; (C) P4HB expression levels in LUAD tissue samples were analyzed using TCGA data; (D) the prognosis of P4HB expression was shown by Kaplan-Meier plotter curves; (E,F) the pathway enrichment of those metabolites with a high P4HB level was predicted; (G) circP4HB expression levels in LUAD tissue samples were detected by RT-qPCR assays; (H,I) the GSH levels and GSH/GSSG ratios of circP4HB overexpression H1299 and SPCA1 cells were measured. ${ }^{* *}, \mathrm{P}<0.01$; ***, $\mathrm{P}<0.001$. LUAD, lung adenocarcinoma; GSH, glutathione; GSSG, oxidized glutathione disulfide.

which SLC7A11 attracted our attention (Figure 3G). SLC7A11 (xCT) is highly expressed in tumor tissues, and is implicated in the ferroptosis of cancer cells $(26,27)$. The dual-luciferase reporter assays used in this study demonstrated that the activity of luciferase decreased when the cells were co-infected with the plasmid (Slc7a11 3'-UTR and miR-1184 mimics) (Figure 3H,3I). These findings indicated that miR-1184 targeted SLC7A11. The knockdown of circP4HB upregulated miR-1184 and downregulated Slc7a11 expression (Figure 37,3K). Thus, 

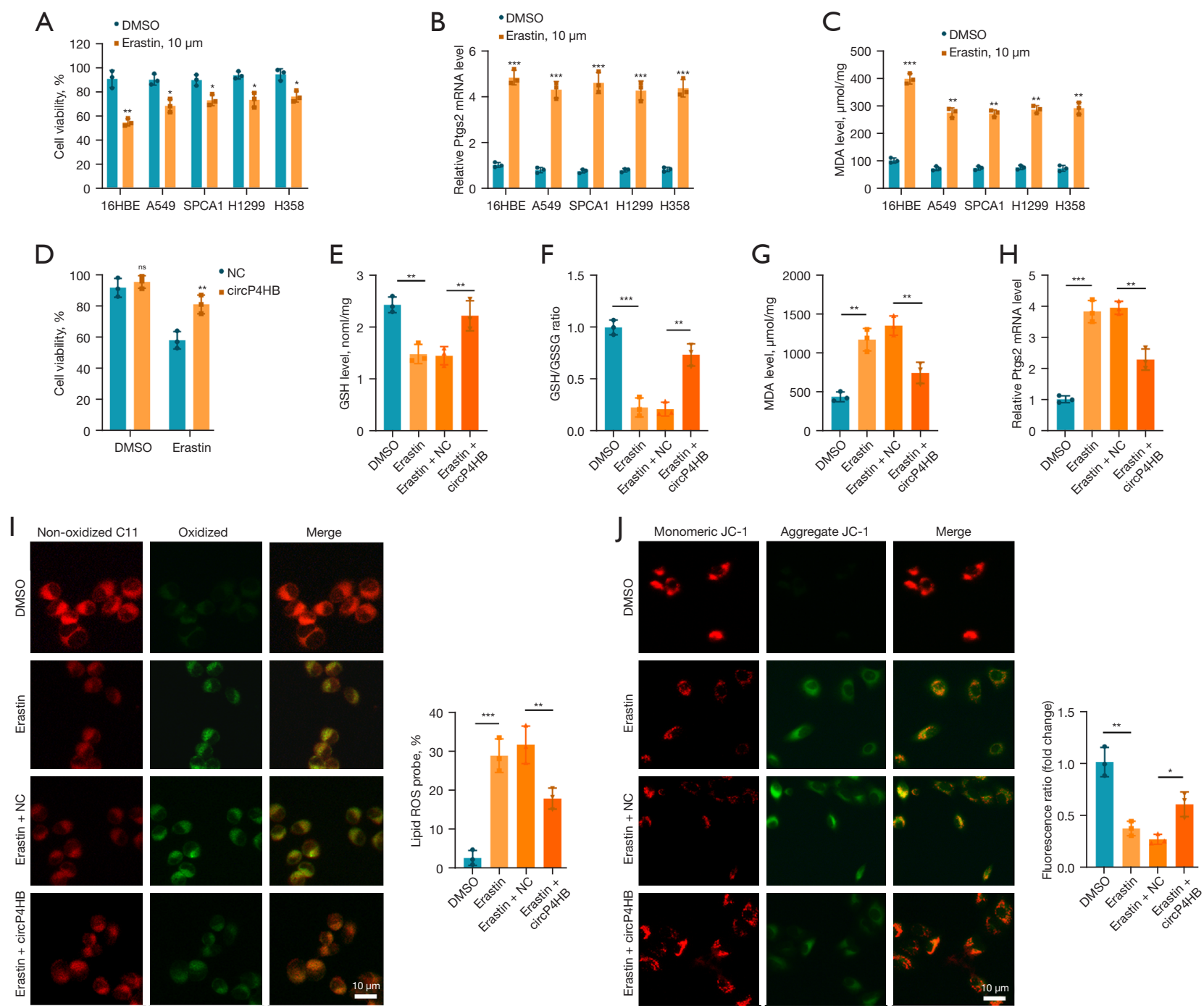

Figure 2 CircP4HB protected LUAD from ferroptosis through promoting GSH synthesis. (A) The effect of erastin (10 $\mu \mathrm{m} 24 \mathrm{~h})$ on the viability of LUAD and 16HBE cells was evaluated by CCK-8. DMSO was used as a control; (B,C) ferroptosis markers, Ptgs2 and MDA, were examined in cell lines in response to erastin $(10 \mu \mathrm{m}, 24 \mathrm{~h})$; (D) the effect of erastin on the viability of H1299 cells overexpressing circP4HB was measured by CCK-8; (E,F) GSH levels and GSH/GSSG ratios of the circP4HB-overexpressing cells were measured; (G,H) Ptgs2 mRNA and MDA levels in circP4HB-overexpressing cells were examined in response to erastin; (I,J) lipid ROS and JC-1 levels were measured following immunofluorescence staining of $\mathrm{H} 1299$ cells following treatment with erastin. * ${ }^{*} \mathrm{P}<0.05 ;{ }^{* *}, \mathrm{P}<0.01 ;{ }^{* * *}, \mathrm{P}<0.001 ; \mathrm{ns}$, non-significant. LUAD, lung adenocarcinoma; Ptgs2, prostaglandin-endoperoxide synthase 2; MDA, malondialdehyde; DMSO, dimethyl sulfoxide; CCK-8, cell counting kit-8; GSH, glutathione; lipid ROS, lipid reactive oxygen species.

circP4HB might function as ceRNA by modulating miR1184 and SLC7A11.

\section{CircP4HB inbibited ferroptosis by regulating miR-1184/ $x C T$-mediated GSH synthesis}

To further explore the regulatory mechanism involved in ferroptosis, a series of rescue experiments were performed. The function of erastin $+\mathrm{LV}$-circP4HB was abolished in response to miR-1184 mimics, and knockdown of Slc7a11 (Figure 4A-4D). Additionally, corresponding changes in SLC7A11 protein levels were detected (Figure 4E). We also examined the mRNA expression of downstream effectors (i.e., Gclc, Gclm, Gsr, Gss, and Nqo1) known to regulating 
A

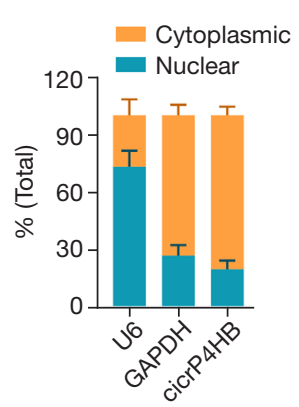

D

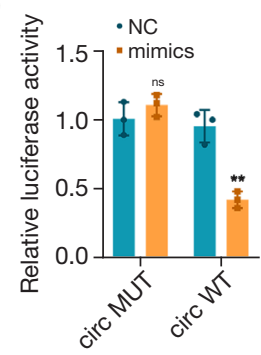

G

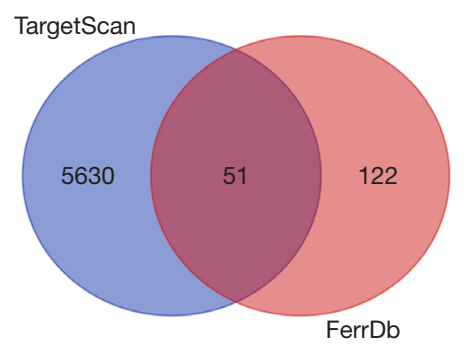

$\mathrm{H}$

\begin{tabular}{|l|l|}
\hline Position 4723-4729 & SLC7A11 3'-UTR \\
\hline
\end{tabular}

SLC7A11 3'UTR-WT 5'-ACCCAAGGGGCAGAAGCUGCAGU-3'

hsa-miR-1184 3'-CCUUCGGUAGUUCAGCGACGUCC-5

SLC7A11 3'UTR-MUT 5'-ACCCAAGGGGCAGAACGACGUCU-3'
$\mathrm{E}$
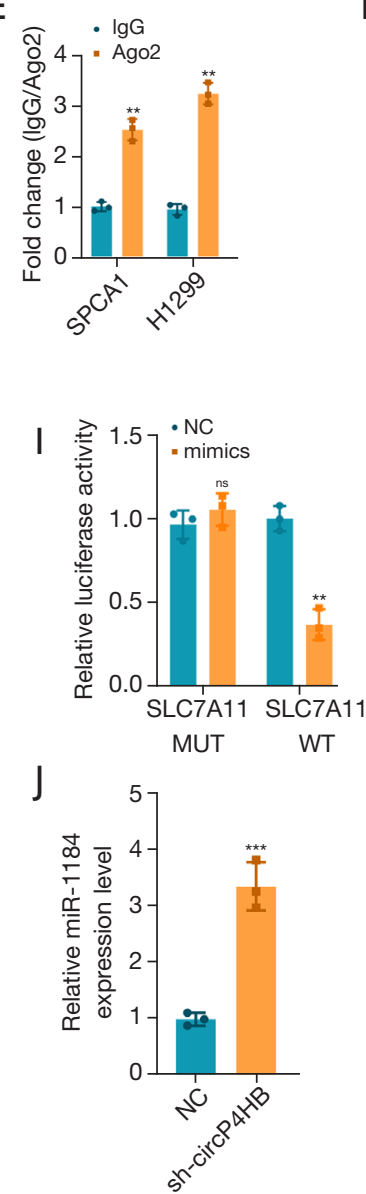

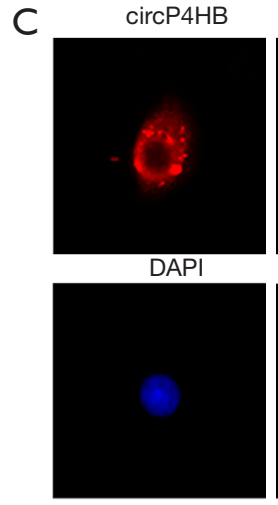

$\mathrm{F}$
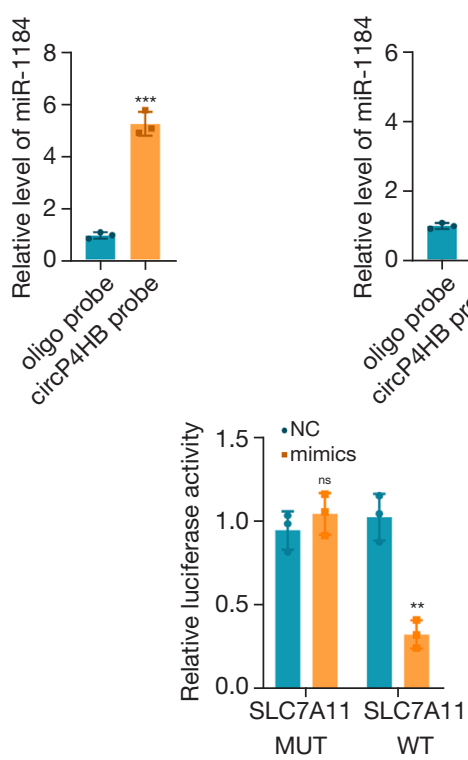

$\mathrm{K}$

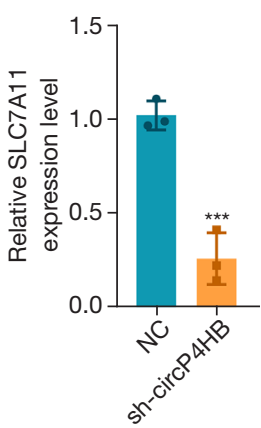

Figure 3 Validation of the interactions between circP4HB, miR-1184, and SLC7A11. (A) The relative abundance of circP4HB in the nucleus and cytoplasm was investigated via nuclear mass separation assays; (B) bioinformatic predictions https://circinteractome.nia.nih. gov and circNet, identified miR-1184 as a target of circP4HB; (C) FISH assay showed circP4HB and miR-1184 probes co-localized in the cytoplasm; (D) the interaction between circP4HB and miR-1184 was determined by using dual-luciferase reporter assays; (E) RIP assays were performed on the cells using AGO2 antibodies; (F) RNA pull-down assays and qRT-PCR assays were used to confirm the binding of circP4HB to miR-1184; (G) predictions using TargetScan and FerrDb, SLC7A11 was taken forward as a target of interest; (H,I) dual-luciferase reporter assays were carried out using HEK-293 T cells co-infected with a plasmid containing the SLC7A11 3'UTR and miR-1184 mimics; (J,K) miR-1184 and SLC7A11 levels were measured in knockdown circP4HB cells. Negative controls (NC) were also used. ${ }^{* *}, \mathrm{P}<0.01$; ${ }^{* *}, \mathrm{P}<0.001$; ns, non-significant. SLC7A11, solute carrier family 7 member 11; AGO2, Argonaute 2; RIP, RNA immunoprecipitation; FISH, fluorescence in situ hybridization. 
A
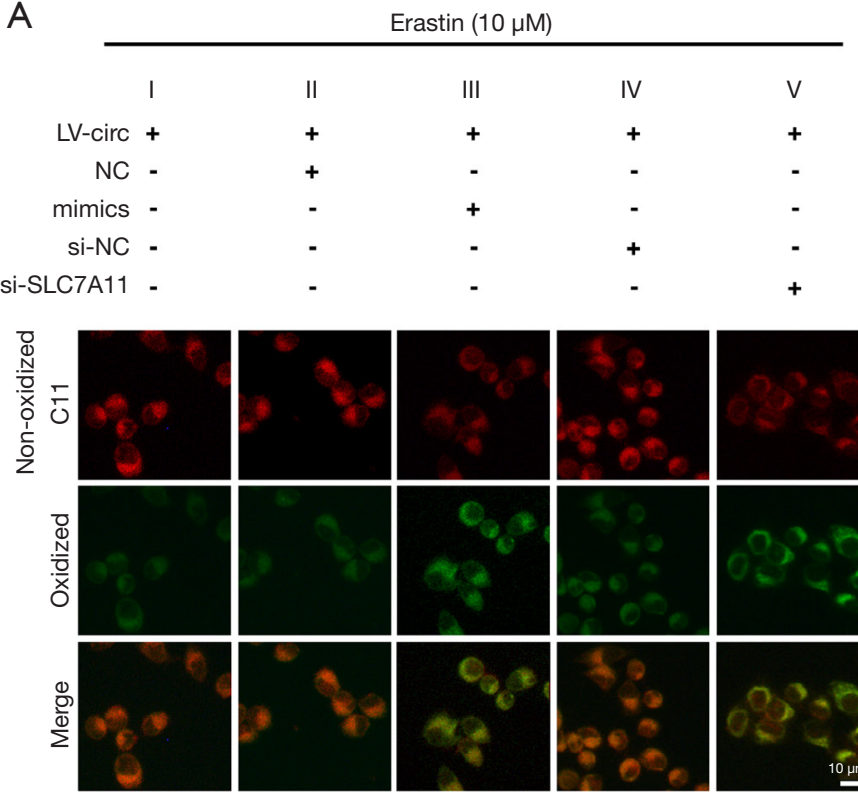

B
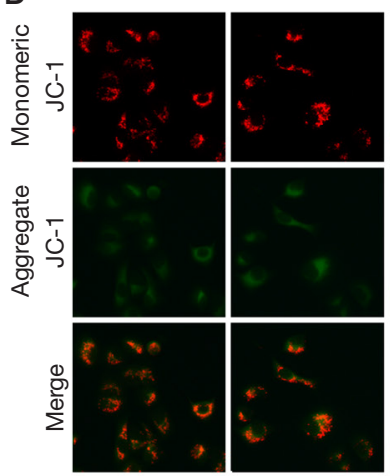
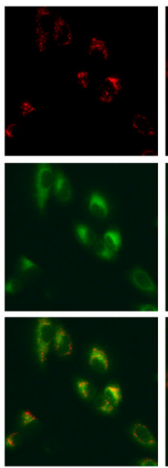
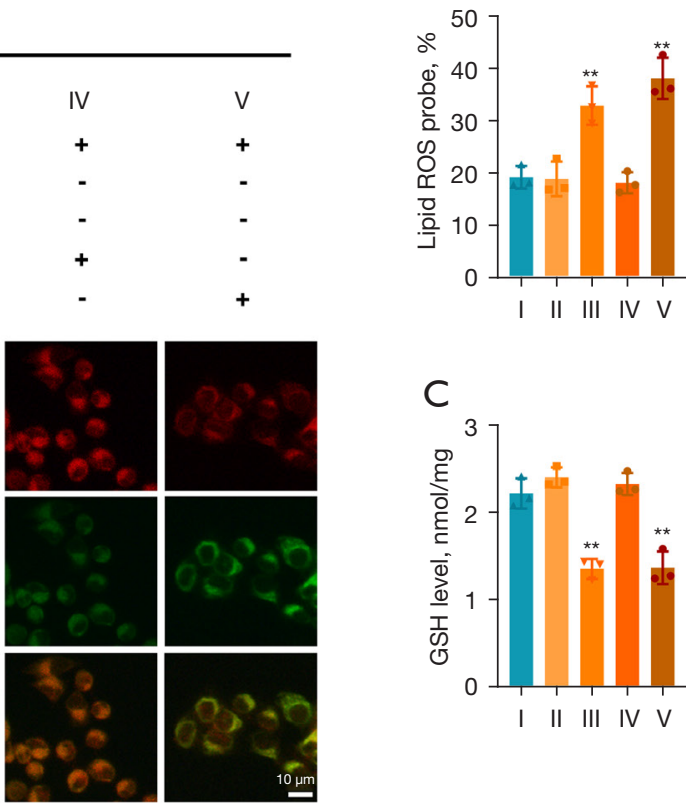

C

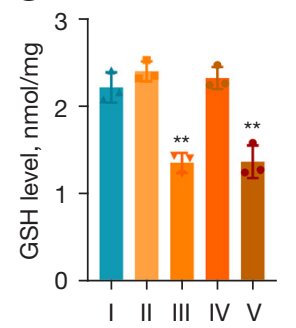

E

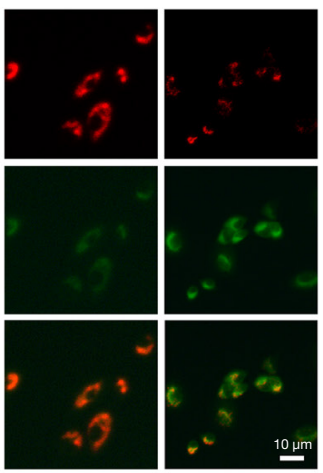

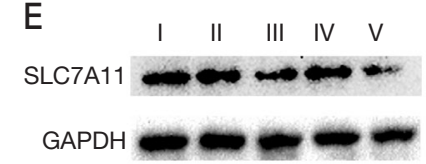

$\mathrm{F}$

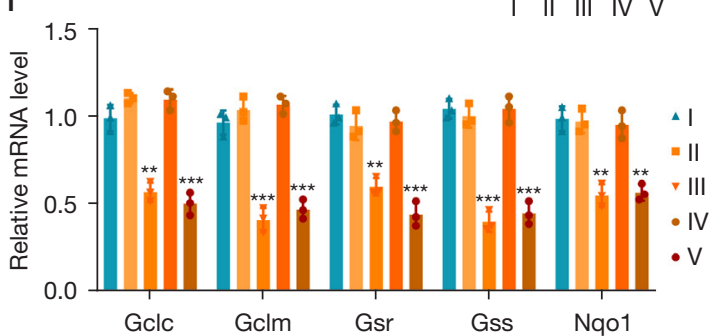

Figure 4 CircP4HB inhibits ferroptosis by regulating miR-1184/SLC7A11-mediated GSH synthesis. (A,B) Lipid ROS and JC-1 levels were measured using Lipid ROS and JC-1 staining; (C,D) GSH levels and the GSH/GSSG ratios were detected; (E) protein levels of SLC7A11 were tested by western blot analysis; (F) mRNA levels of downstream effectors (Gclc, Gclm, Gsr, Gss, and Nqo1) were examined by qRTPCR. +, transfected with; -, transfected without. ${ }^{* *}, \mathrm{P}<0.01$; ${ }^{* * *}, \mathrm{P}<0.001$. SLC7A11, solute carrier family 7 member 11; GSH, glutathione; lipid ROS, lipid reactive oxygen species; GSSG, oxidized glutathione disulfide.

GSH metabolism, and found that the increased mRNA level of erastin + LV-circP4HB was abolished by miR1184 mimics, and the knockdown of Slc7a11 (Figure 4F). These data highlight important findings that that circP4HB inhibits ferroptosis by regulating the miR-1184/SLC7A11 axis that mediates GSH synthesis.

\section{CircP4HB promoted LUAD growth and inbibited ferroptosis in vivo}

Mice injected with circP4HB-overexpressing cells had larger tumors with corresponding tumor weights and volumes which were significantly greater in mice treated with transfected H1299 cells overexpressing circP4HB than 
controls (Figure 5A-5C). The expression levels of circ $\mathrm{P} 4 \mathrm{HB}$ and Slc7a11 mRNA were increased in the mouse tumors in response to injection with cells overexpressing circP4HB (Figure 5D,5E). Additionally, the immunohistochemistry (IHC) assays showed that the SLC7A11 levels were higher in the experimental overexpressing circP4HB groups than the relative negative control groups (Figure $5 F$ ).

TCGA data showed that Slc7a11 mRNA expression was higher in LUAD tissues relative to normal lung tissue (Figure 5G). Kaplan-Meier plotter analysis indicated that patients with high Slc7a11 expression levels have significantly poorer overall survival (see Figure $5 H$ ). In a cohort of 50 LUAD patients, Slc7a11 mRNA was significantly higher in tumor tissues compared to corresponding para-tumor tissues (Figure 5I). Furthermore, IHC staining supported this observation in SLC7A11 expression, where this was increased in LUAD tissues (Figure 57).

\section{Discussion}

Here we provide interesting insights into the molecular mechanisms underlying LUAD ferroptosis by identifying a novel pathway. CircP $4 \mathrm{HB}$ protects LUAD from ferroptosis via SLC7A11-mediated GSH synthesis. A model summarizing these concepts is displayed in Figure 6. The discovery of ferroptosis has opened up a new platform in the field of cancer research, and its clinical significance in cancer development has gradually emerged. The question of how to intervene in the development of cancers by regulating cell ferroptosis is a popular research area. Targeting ferroptosis (including ferroptosis inducers such as small molecules and nanomaterials) may become a new strategy for the treatment of cancers.

CircRNA cIARS modulate ferroptosis in hepatic carcinoma by interacting with ALKBH5 (28). In lung cancer, circDTL functions as an oncogene and contributes to cell ferroptosis (29). Both Li et al. and Wang et al. examined the function of circP4HB in lung cancer metastasis $(30,31)$. However, its role in LUAD proliferation and ferroptosis is unclear. We identified circ $\mathrm{P} 4 \mathrm{HB}$ as a novel ferroptosis suppressor circRNA, and found that circP4HB promoted LUAD growth and inhibited ferroptosis both in vitro and in vivo.

Ferroptosis is a non-apoptotic form of cell death caused by the accumulation of toxic lipid peroxidation, and the accumulation of intracellular ROS is one of the direct causes of ferroptosis (32). It shows that lung cancer tissues generally have higher levels of reactive oxygen species and ROS, indicating ferroptosis is involved in the occurrence and development of lung cancer. As key regulators of ferroptosis, SLC7A11 and GSH can be regulated by a variety of upstream factors including lncRNAs and circRNAs, and are involved in the progression of lung adenocarcinoma (33). For example, circRNA_101093 regulates SLC7A11 and GSH to alter the ferroptosis desensitization of lung adenocarcinoma (34). We found that circP4HB could inhibit ferroptosis in lung adenocarcinoma, and this effect is achieved by increasing the expression of Slc7a11, which may provide information for the regulation of LUAD ferroptosis a new mechanism.

GSH plays an essential role in regulation of ferroptosis, and participates in cancer progression (13). Based on GSH metabolism pathway enrichment in high P4HB (the host gene of circ_0046263), and the positive expression, we speculated that circP4HB might be related to GSH metabolism. To test this hypothesis, we detected the endproduct of GSH metabolism, and found that GSH was significantly upregulated in circP4HB-overexpressing cells. Further, we found that circP4HB protected LUAD from ferroptosis via GSH synthesis. Many ferroptosis pathways have been established, but the regulatory network remains unclear.

CeRNAs is a complicated mechanism involving in the interactions between multiple RNAs, which contribute to various biological processes $(35,36)$. There is increasing evidence that both circRNAs and lncRNAs function as an miRNA sponge via the ceRNA mechanism in cancer $(37,38)$. In hepatic carcinoma, circNFATC3 sponges miR-548I, and functions as a ceRNA to regulate cancer progression (39). In pancreatic ductal adenocarcinoma, circ-0005105 modulates COL11A1 by targeting miR-20a-3p to regulate cancer progression (40). CircP4HB has been reported to be a ceRNA that affects the development of nasopharyngeal carcinoma (41). We demonstrated that circP4HB sponged miR-1184, and miR-1184 targeted SLC7A11. Collectively, circP4HB might function as a ceRNA by modulating miR1184 and SLC7A11. Knocking down circP4HB upregulated miR-1184 and downregulated SLC7A11.

SLC7A11 (xCT) was highly expressed at the cytoplasmic membrane, and SLC7A11-mediated metabolic reprogramming promotes lung cancer progression (42). The suppression of the SLC7A11/GSH axis causes synthetic lethality in KRAS-mutant LUAD (43). We re-analyzed TCGA data, and found that SLC7A11 mRNA level was higher in LUAD tissues, and patients with high Slc7a11 


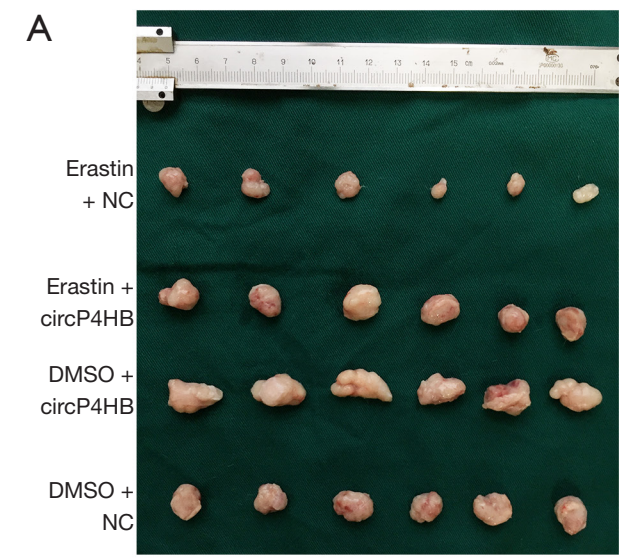

B

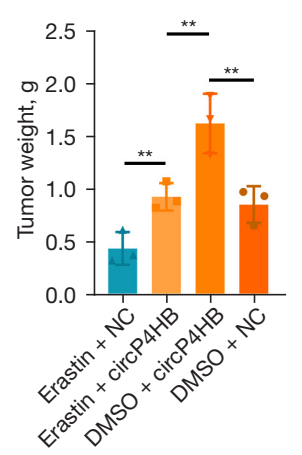

F

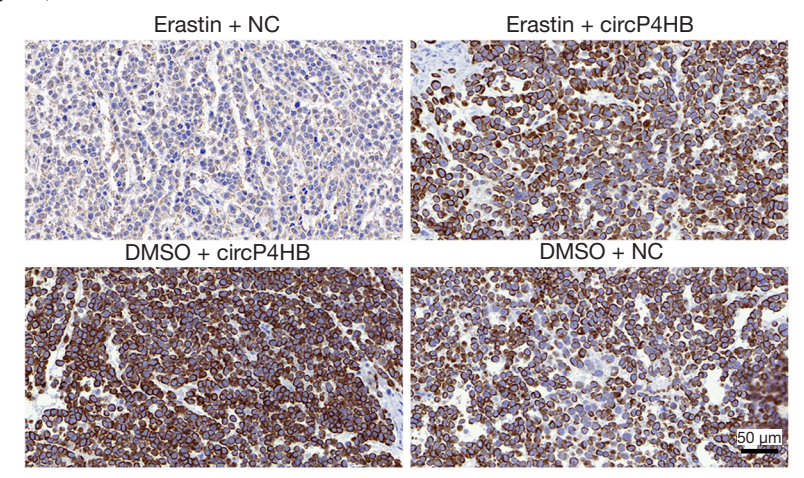

C

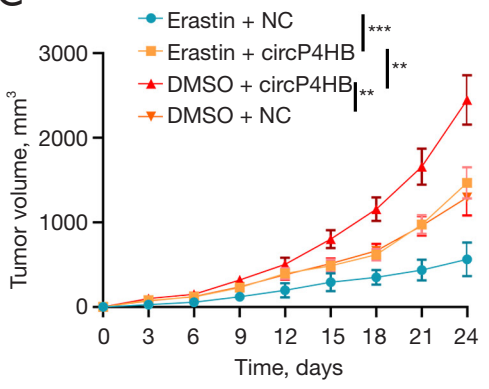

D

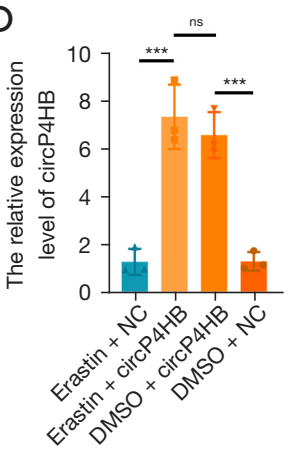

G

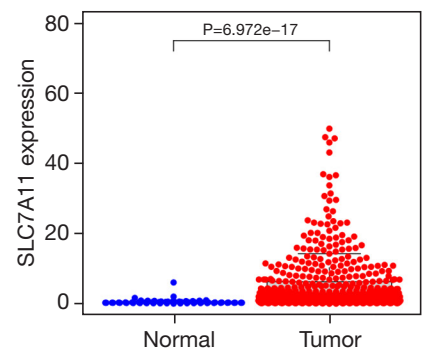

I

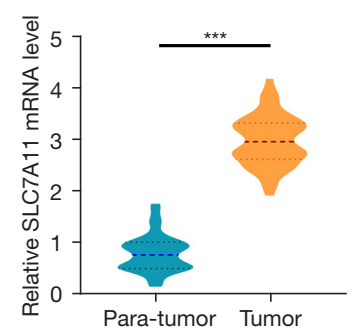

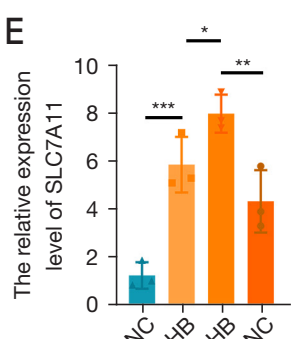
$\therefore 0$

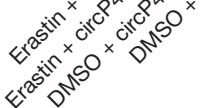
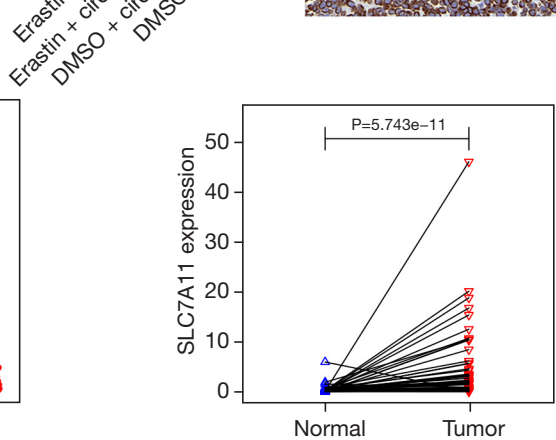

$\mathrm{H}$

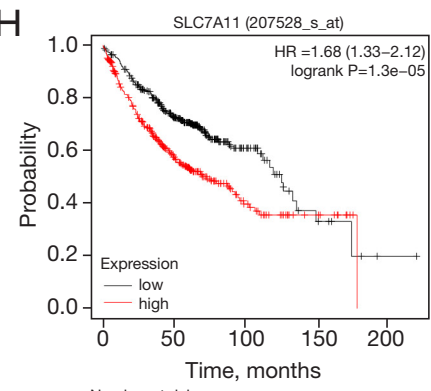

J \begin{tabular}{lllll}
\multicolumn{5}{c}{ Number at risk } \\
low 368 & 207 & 38 & 9 & 1 \\
high 351 & 140 & 31 & 10 & 0
\end{tabular}

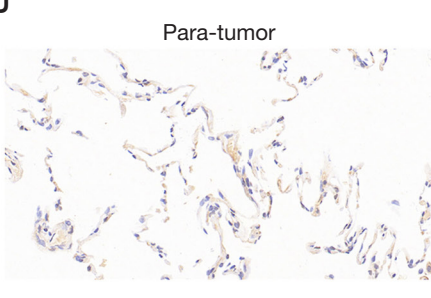




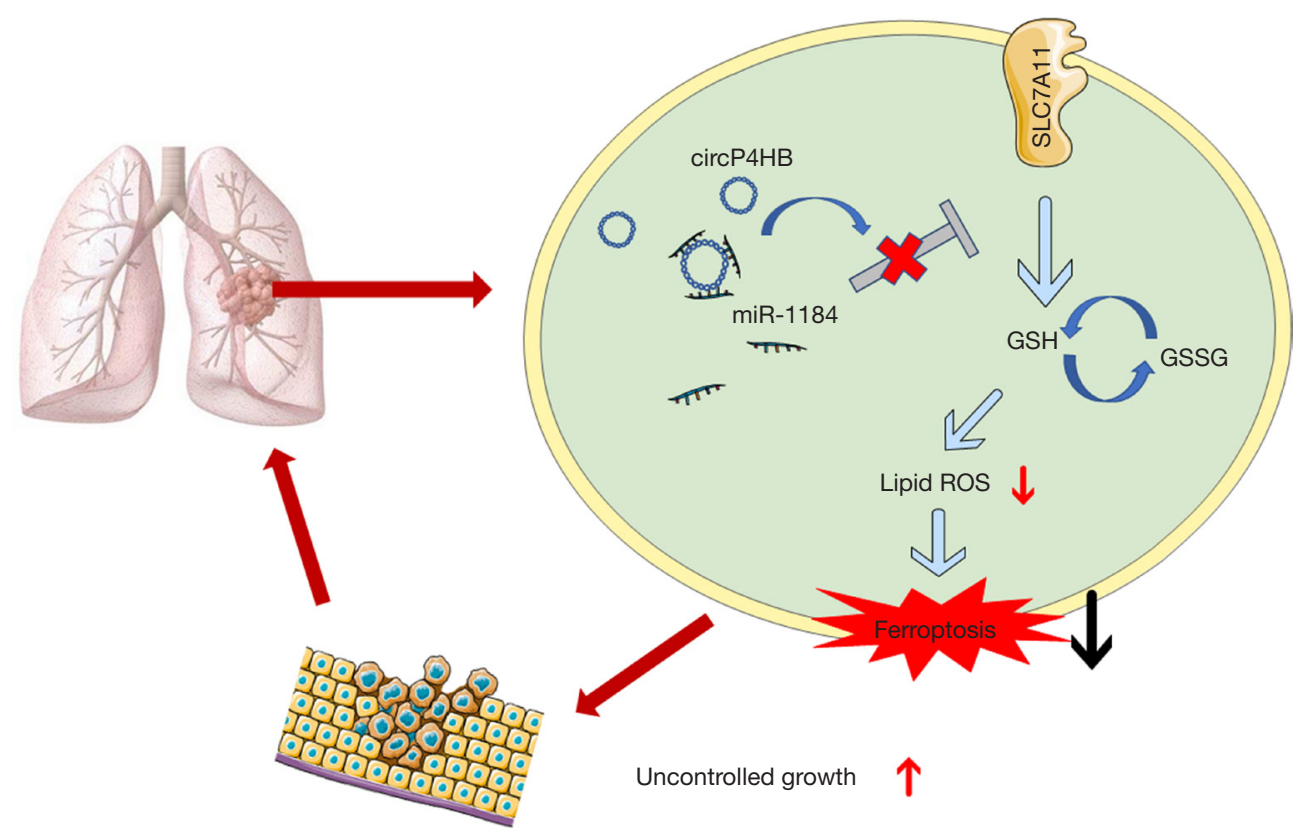

Figure 6 Schematic representation of a proposed mechanism by which circP4HB protects lung adenocarcinoma cells from ferroptosis via SLC7A11-mediated glutathione synthesis. SLC7A11, solute carrier family 7 member 11; GSH, glutathione; GSSG, oxidized glutathione disulfide; ROS, reactive oxygen species.

expression levels had significantly poorer overall survival. Additionally, we found that Slc7a11 was significantly higher in tumor tissues than in para-tumor tissues in 50 cases.

This work sought to show that $\operatorname{circP} 4 \mathrm{HB}$ is a key regulator of ferroptosis in LUAD. Notably, we demonstrated the following: (I) circP4HB triggers GSH synthesis; (II) circP4HB inhibits ferroptosis in LUAD; (III) circP4HB acts as a "miRNA sponge" to relieve the inhibitory effect of miR-1184 on SLC7A11, which functions as ceRNA; and (IV) circP4HB /miR-1184/ SLC7A11axis protects LUAD from ferroptosis. In light of these findings, this study had several limitations. First, there are many ways to regulate ferroptosis, such as SLC7A11-mediated GSH, GPX4, and ACSL4. In this study, we only demonstrated the role of circP4HB in ferroptosis via SLC7A11-mediated GSH. Second, the effect of circP4HB, SLC7A11 and GSH metabolism on LUAD prognosis needs to be further explored. Given the evidence on the roles of ferroptosis in cancer, it is important to investigate its mechanism, which still remains elusive.

In summary, findings from this study identified circP4HB as a novel ferroptosis suppressor in LUAD which promotes tumor growth and protects tumor cells from undergoing ferroptosis via the miR-1184/SLC7A11 axis. Ferroptosis provides a new avenue for the treatment of lung cancer. Clinically, some drugs and radiation therapy have been found to induce cell ferroptosis and drugs targeting RNA molecules has also received great attention (44). Our study provides circP4HB as a site for molecule-targeted therapy, which also provides a new research direction for ferroptosis-inducing therapy in lung adenocarcinoma. In addition to adding further knowledge to the role of circ $\mathrm{P} 4 \mathrm{HB}$ in regulating ferroptosis in lung cancer, these data highlight the prognostic and therapeutic potential of the circRNA, circP4HB, as a biomarker in LUAD. Further studies are warranted to further validate these findings in a larger cohort of lung cancer patients and indeed, other lung cancer histologies.

\section{Acknowledgments}

The authors appreciate the academic support from the AME Thoracic Surgery Collaborative Group.

Funding: This work was supported by the National Natural Science Foundation of China (No. 81902328), the Key Medical Research Project of Jiangsu Provincial Health Commission (No. K2019002), the Ethicon Excellence in 
Surgery Grant (No. HZB 20190528-13), and the Jiangsu Province Natural Science Foundation (No. BK20201492).

\section{Footnote}

Reporting Checklist: The authors have completed the ARRIVE reporting checklist. Available at https://tlcr. amegroups.com/article/view/10.21037/tlcr-22-138/rc

Data Sharing Statement: Available at https://tlcr.amegroups. com/article/view/10.21037/tlcr-22-138/dss

Conflicts of Interest: All authors have completed the ICMJE uniform disclosure form (available at https://tlcr.amegroups. com/article/view/10.21037/tlcr-22-138/coif). The authors have no conflicts of interests to declare.

Ethical Statement: The authors are accountable for all aspects of the work, including ensuring that any questions related to the accuracy or integrity of any part of the work have been appropriately investigated and resolved. The present study was approved by the Institutional Ethics Committee of the First Affiliated Hospital with Nanjing Medical University (No. 2019-SFRA-082), and written informed consent was obtained from each participant. The study was conducted in accordance with the Declaration of Helsinki (as revised in 2013). The animal experiments were performed according to the guidelines of the Institutional Animal Care and Use Committee of Nanjing Medical University (Nanjing, China) and protocols were approved by the Institutional Animal Care and Use Committee of Nanjing Medical University (No. IACUC:2009054).

Open Access Statement: This is an Open Access article distributed in accordance with the Creative Commons Attribution-NonCommercial-NoDerivs 4.0 International License (CC BY-NC-ND 4.0), which permits the noncommercial replication and distribution of the article with the strict proviso that no changes or edits are made and the original work is properly cited (including links to both the formal publication through the relevant DOI and the license). See: https://creativecommons.org/licenses/by-nc-nd/4.0/.

\section{References}

1. Siegel RL, Miller KD, Jemal A. Cancer statistics, 2020. CA Cancer J Clin 2020;70:7-30.

2. Strano S, Lupo A, Lococo F, et al. Prognostic significance of vascular and lymphatic emboli in resected pulmonary adenocarcinoma. Ann Thorac Surg 2013;95:1204-10.

3. Travis WD, Asamura H, Bankier AA, et al. The IASLC Lung Cancer Staging Project: Proposals for Coding T Categories for Subsolid Nodules and Assessment of Tumor Size in Part-Solid Tumors in the Forthcoming Eighth Edition of the TNM Classification of Lung Cancer. J Thorac Oncol 2016;11:1204-23.

4. Chen Y, Li N, Wang H, et al. Amentoflavone suppresses cell proliferation and induces cell death through triggering autophagy-dependent ferroptosis in human glioma. Life Sci 2020;247:117425.

5. Su Y, Zhao B, Zhou L, et al. Ferroptosis, a novel pharmacological mechanism of anti-cancer drugs. Cancer Lett 2020;483:127-36.

6. Yang WS, SriRamaratnam R, Welsch ME, et al. Regulation of ferroptotic cancer cell death by GPX4. Cell 2014;156:317-31.

7. Ye Z, Zhuo Q, Hu Q, et al. FBW7-NRA41-SCD1 axis synchronously regulates apoptosis and ferroptosis in pancreatic cancer cells. Redox Biol 2021;38:101807.

8. Li J, Cao F, Yin HL, et al. Ferroptosis: past, present and future. Cell Death Dis 2020;11:88.

9. Mou Y, Wang J, Wu J, et al. Ferroptosis, a new form of cell death: opportunities and challenges in cancer. J Hematol Oncol 2019;12:34.

10. Tang B, Zhu J, Li J, et al. The ferroptosis and ironmetabolism signature robustly predicts clinical diagnosis, prognosis and immune microenvironment for hepatocellular carcinoma. Cell Commun Signal 2020;18:174.

11. Li D, Li Y. The interaction between ferroptosis and lipid metabolism in cancer. Signal Transduct Target Ther 2020;5:108.

12. Zhu J, Xiong Y, Zhang Y, et al. The Molecular Mechanisms of Regulating Oxidative Stress-Induced Ferroptosis and Therapeutic Strategy in Tumors. Oxid Med Cell Longev 2020;2020:8810785.

13. Sun Y, Zheng Y, Wang C, et al. Glutathione depletion induces ferroptosis, autophagy, and premature cell senescence in retinal pigment epithelial cells. Cell Death Dis 2018;9:753.

14. Wang J, Zhao X, Wang Y, et al. circRNA-002178 act as a ceRNA to promote PDL1/PD1 expression in lung adenocarcinoma. Cell Death Dis 2020;11:32.

15. Liu Z, Zhou Y, Liang G, et al. Circular RNA hsa_ circ_001783 regulates breast cancer progression via sponging miR-200c-3p. Cell Death Dis 2019;10:55. 
16. Zheng H, Shi L, Tong C, et al. circSnx12 Is Involved in Ferroptosis During Heart Failure by Targeting miR-2245p. Front Cardiovasc Med 2021;8:656093.

17. Xia Y, Wei K, Yang FM, et al. miR-1260b, mediated by YY1, activates KIT signaling by targeting SOCS6 to regulate cell proliferation and apoptosis in NSCLC. Cell Death Dis 2019;10:112.

18. Lv X, Huang H, Feng H, et al. Circ-MMP2 (circ-0039411) induced by FOXM1 promotes the proliferation and migration of lung adenocarcinoma cells in vitro and in vivo. Cell Death Dis 2020;11:426.

19. Xu L, Liao WL, Lu QJ, et al. Hypoxic tumorderived exosomal circular RNA SETDB1 promotes invasive growth and EMT via the miR-7/Sp1 axis in lung adenocarcinoma. Mol Ther Nucleic Acids 2021;23:1078-92.

20. Yu Z, Zhu X, Li Y, et al. Circ-HMGA2 (hsa_circ_0027446) promotes the metastasis and epithelial-mesenchymal transition of lung adenocarcinoma cells through the miR1236-3p/ZEB1 axis. Cell Death Dis 2021;12:313.

21. Zhou J, Zhang S, Chen Z, et al. CircRNA-ENO1 promoted glycolysis and tumor progression in lung adenocarcinoma through upregulating its host gene ENO1. Cell Death Dis 2019;10:885.

22. Kalinina EV, Gavriliuk LA. Glutathione Synthesis in Cancer Cells. Biochemistry (Mosc) 2020;85:895-907.

23. Chen Y, Zhu G, Liu Y, et al. O-GlcNAcylated c-Jun antagonizes ferroptosis via inhibiting GSH synthesis in liver cancer. Cell Signal 2019;63:109384.

24. Li X, Ding J, Wang X, et al. NUDT21 regulates circRNA cyclization and ceRNA crosstalk in hepatocellular carcinoma. Oncogene 2020;39:891-904.

25. Sheu-Gruttadauria J, Xiao Y, Gebert LF, et al. Beyond the seed: structural basis for supplementary microRNA targeting by human Argonaute2. EMBO J 2019;38:e101153.

26. Ghoochani A, Hsu EC, Aslan M, et al. Ferroptosis Inducers Are a Novel Therapeutic Approach for Advanced Prostate Cancer. Cancer Res 2021;81:1583-94.

27. Xu X, Zhang X, Wei C, et al. Targeting SLC7A11 specifically suppresses the progression of colorectal cancer stem cells via inducing ferroptosis. Eur J Pharm Sci 2020;152:105450.

28. Liu Z, Wang Q, Wang X, et al. Circular RNA cIARS regulates ferroptosis in $\mathrm{HCC}$ cells through interacting with RNA binding protein ALKBH5. Cell Death Discov 2020;6:72.

29. Shanshan $W$, Hongying $M$, Jingjing F, et al. CircDTL
Functions as an Oncogene and Regulates Both Apoptosis and Ferroptosis in Non-small Cell Lung Cancer Cells. Front Genet 2021;12:743505.

30. Li G, Zhao C, Zhang H, et al. Hsa_circ_0046263 Drives the Carcinogenesis and Metastasis of Non-Small Cell Lung Cancer Through the Promotion of NOVA2 by Absorbing Mir-940 as a Molecular Sponge. Cancer Manag Res 2020;12:12779-90.

31. Wang T, Wang X, Du Q, et al. The circRNA circP4HB promotes NSCLC aggressiveness and metastasis by sponging miR-133a-5p. Biochem Biophys Res Commun 2019;513:904-11.

32. Hirschhorn T, Stockwell BR. The development of the concept of ferroptosis. Free Radic Biol Med 2019;133:130-43.

33. Wu S, Zhu C, Tang D, et al. The role of ferroptosis in lung cancer. Biomark Res 2021;9:82.

34. Zhang X, Xu Y, Ma L, et al. Essential roles of exosome and circRNA_101093 on ferroptosis desensitization in lung adenocarcinoma. Cancer Commun (Lond) 2022. [Epub ahead of print].

35. Li LJ, Zhao W, Tao SS, et al. Competitive endogenous RNA network: potential implication for systemic lupus erythematosus. Expert Opin Ther Targets 2017;21:639-48.

36. Karreth FA, Pandolfi PP. ceRNA cross-talk in cancer: when ce-bling rivalries go awry. Cancer Discov 2013;3:1113-21.

37. Yang J, Cheng M, Gu B, et al. CircRNA_09505 aggravates inflammation and joint damage in collagen-induced arthritis mice via miR-6089/AKT1/NF-kappaB axis. Cell Death Dis 2020;11:833.

38. Kong X, Duan Y, Sang Y, et al. LncRNA-CDC6 promotes breast cancer progression and function as ceRNA to target CDC6 by sponging microRNA-215. J Cell Physiol 2019;234:9105-17.

39. Jia C, Yao Z, Lin Z, et al. circNFATC3 sponges miR-548I acts as a ceRNA to protect NFATC3 itself and suppressed hepatocellular carcinoma progression. J Cell Physiol 2021;236:1252-69.

40. Ma G, Li G, Fan W, et al. Circ-0005105 activates COL11A1 by targeting miR-20a-3p to promote pancreatic ductal adenocarcinoma progression. Cell Death Dis 2021;12:656.

41. Yin L, Chen J, Ma C, et al. Hsa_circ_0046263 functions as a ceRNA to promote nasopharyngeal carcinoma progression by upregulating IGFBP3. Cell Death Dis 2020;11:562.

42. Ji X, Qian J, Rahman SMJ, et al. xCT (SLC7A11)-mediated metabolic reprogramming promotes non-small cell lung 
cancer progression. Oncogene 2018;37:5007-19.

43. Hu K, Li K, Lv J, et al. Suppression of the SLC7A11/ glutathione axis causes synthetic lethality in KRAS-mutant lung adenocarcinoma. J Clin Invest 2020;130:1752-66.

Cite this article as: Pan CF, Wei K, Ma ZJ, He YZ, Huang JJ, Guo ZZ, Chen ZP, Barr MP, Shackelford RE, Xia Y, Wang J. CircP4HB regulates ferroptosis via SLC7A11-mediated glutathione synthesis in lung adenocarcinoma. Transl Lung Cancer Res 2022;11(3):366-380. doi: 10.21037/tlcr-22-138
44. Xiong R, He R, Liu B, et al. Ferroptosis: A New Promising Target for Lung Cancer Therapy. Oxid Med Cell Longev 2021;2021:8457521. 
A
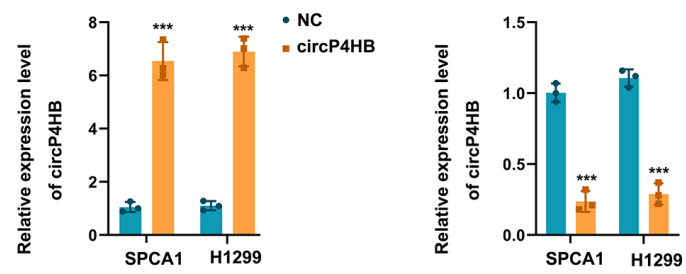

B

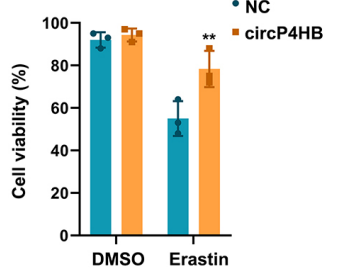

C
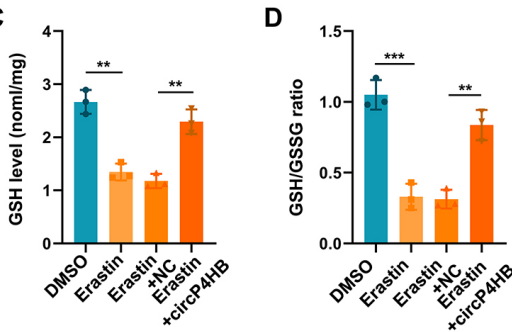

E

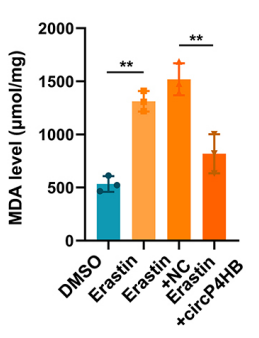

F

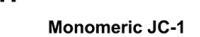

Aggregate JC-1 Merge
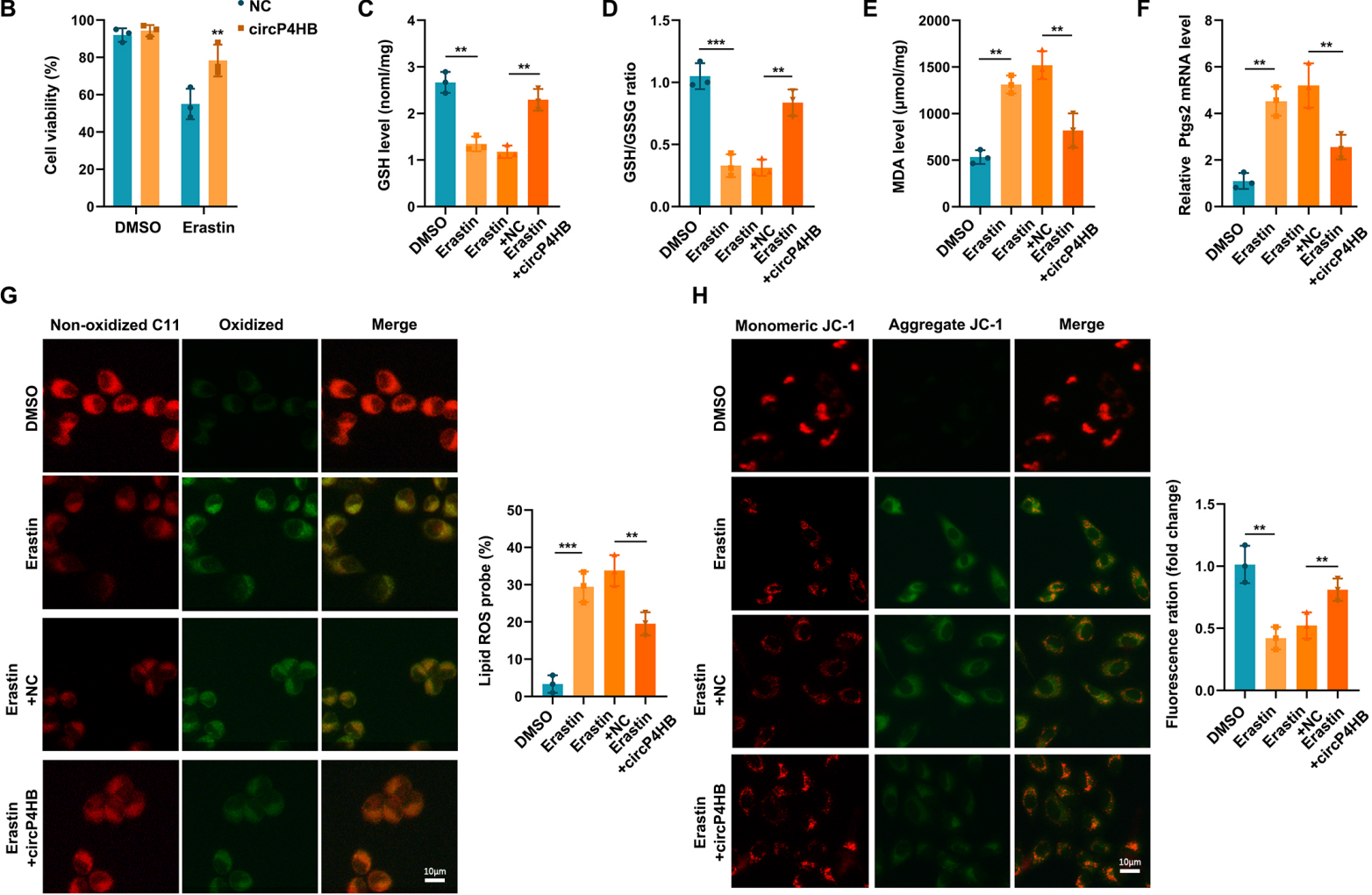

I

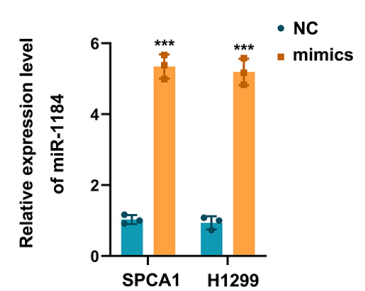

$\mathbf{J}$

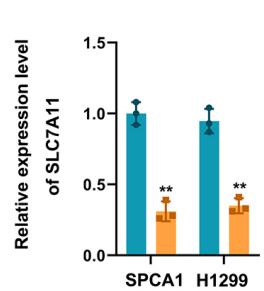

Figure S1 CircP4HB protects LUAD SPCA1 cells from ferroptosis by promoting GSH synthesis. (A) Transfection efficiency was determined in both SPCA1 and H1299 cell lines by qRT-PCR; (B) cell viability was assessed using the CCK8 assay; (C,D) the GSH levels and GSH/GSSG ratios of circP4HB-overexpressing cells were detected; (E,F) the MDA levels and Ptgs2 gene levels in circP4HBoverexpressing cells following treatment with erastin and DMSO controls were tested; $(\mathrm{G}, \mathrm{H})$ the lipid ROS and JC-1 levels of treated SPCA1 were measured; (I,J) transfection efficiency of miR-1184 or SLC7A11 was determined in SPCA1 and H1299 cell lines via by qRTPCR respectively. ${ }^{* *} \mathrm{P}<0.01 ;{ }^{* *} \mathrm{P}<0.001$. LUAD, lung adenocarcinoma; GSH, glutathione; GSSG, oxidized glutathione disulfide; MDA, malondialdehyde; Ptgs2, prostaglandin-endoperoxide synthase 2; DMSO, dimethyl sulfoxide; SLC7A11, solute carrier family 7 member 11. 